\title{
Prior beta blocker treatment decreases leukocyte responsiveness to injury
}

\author{
Laurel A. Grisanti, ${ }^{1}$ Claudio de Lucia, ${ }^{2}$ Toby P. Thomas, ${ }^{2}$ Aron Stark, ${ }^{3}$ John T. Strony, ${ }^{3}$ \\ Valerie D. Myers, ${ }^{4}$ Remus Beretta, ${ }^{4}$ Daohai Yu, ${ }^{5}$ Celestino Sardu, ${ }^{6}$ Raffaele Marfella, ${ }^{6}$ Erhe Gao, ${ }^{2}$ \\ Steven R. Houser, ${ }^{4}$ Walter J. Koch, ${ }^{2}$ Eman A. Hamad, ${ }^{3}$ and Douglas G. Tilley ${ }^{2}$ \\ 'Department of Biomedical Sciences, College of Veterinary Medicine, University of Missouri, Columbia, Missouri, USA. \\ ${ }^{2}$ Center for Translational Medicine, ${ }^{3}$ Department of Medicine, ${ }^{4}$ Center for Cardiovascular Research, and ${ }^{5}$ Department of \\ Clinical Sciences, Lewis Katz School of Medicine, Temple University, Philadelphia, Pennsylvania, USA. ${ }^{6}$ Department of \\ Medical, Surgical, Neurological, Metabolic and Aging Sciences, University of Campania “Luigi Vanvitelli," \\ Piazza Miraglia, 2, Naples, Italy.
}

Following injury, leukocytes are released from hematopoietic organs and migrate to the site of damage to regulate tissue inflammation and repair; however, leukocytes lacking $\boldsymbol{\beta}_{2}$-adrenergic receptor ( $\beta_{2}$-AR) expression have marked impairments in these processes. Beta blockade is a common strategy for the treatment of many cardiovascular etiologies; therefore, the objective of our study was to assess the impact of prior beta blocker treatment on baseline leukocyte parameters and their responsiveness to acute injury. In a temporal and $\beta$-adrenergic receptor isoform-dependent manner, chronic beta blocker infusion increased splenic vascular cell adhesion molecule 1 expression and leukocyte accumulation (monocytes/macrophages, mast cells, and neutrophils) and decreased chemokine receptor 2 (CCR2) expression and migration of bone marrow and peripheral blood leukocytes (PBLs) to, as well as infiltration into, the heart following acute cardiac injury. Further, CCR2 expression and migratory responsiveness were significantly reduced in the PBLs of patients receiving beta blocker therapy compared with beta blocker-naive patients. These results highlight the ability of chronic beta blocker treatment to alter baseline leukocyte characteristics that decrease leukocytes' responsiveness to acute injury and suggest that prior beta blockade may act to reduce the severity of innate immune responses.

Conflict of interest: The authors have declared that no conflict of interest exists.

Copyright: (c) 2019 American Society for Clinical Investigation

Submitted: December 26, 2017 Accepted: March 21, 2019 Published: May 2, 2019.

Reference information: /CI Insight. 2019;4(9):e99485. https://doi. org/10.1172/jici.nsight.99485.

\section{Introduction}

Leukocytes are rapidly recruited to sites of acute injury, where they regulate a wide variety of remodeling responses, including inflammation, fibrosis, and scar stabilization, to promote wound healing (1-4). This early immune response includes the recruitment of inflammatory leukocytes, including granulocytes such as neutrophils and mast cells, as well as phagocytic monocytes, to attract further leukocyte recruitment and begin clearing the area of dead cells and debris $(5,6)$. Intense early or prolonged recruitment of inflammatory leukocytes can lead to maladaptive tissue remodeling or scar formation over time, with a negative impact on organ function, such as increased stiffness that reduces cardiac output following myocardial infarction (MI) $(7,8)$. Dampening leukocyte infiltration following injury has been shown capable of reducing subsequent maladaptive remodeling outcomes (8-12). Therefore, identifying therapeutics that reduce leukocyte responsiveness to acute injury is of great interest.

Acute injury events activate the sympathetic nervous system to regulate a host of immune system functions in part via activation of $\beta$-adrenergic receptors ( $\beta$-ARs) (13). Therefore, understanding whether $\beta$-AR-mediated regulation of immune cells influences their response to injury may offer insight into how this process may be modulated. To this end, we previously developed chimeric mice lacking $\beta_{2}$-AR expression in cells of hematopoietic origin via transplantation of $\beta_{2}$-AR-knockout $\left(\beta_{2}\right.$ $\mathrm{AR}-\mathrm{KO}$ ) bone marrow (BM) into wild-type (WT, C57BL/6J) recipient mice and subjected them to $\mathrm{MI}$. Strikingly, these chimeric mice displayed severe changes in several leukocyte parameters, including increased splenic vascular cell adhesion molecule 1 (VCAM-1) expression and leukocyte retention, as well as decreased bone marrow cell (BMC) expression of chemokine receptor 2 (CCR2) and leukocyte recruitment to the heart after injury $(14,15)$. Collectively, these genetic studies suggested that because 
complete $\beta_{2}$-AR deletion negatively regulates leukocyte behavior both at baseline and following injury, clinically used beta blockers may also influence leukocyte responsiveness to acute injury.

Beta blockers have been used successfully for decades to treat several pathologies, including hypertension, congestive heart failure, and post-MI dysfunction (16). Many of the benefits of beta blockers are thought to be mediated via their direct actions on cardiac-expressed $\beta$-AR to decrease cardiac output when administered after an event such as MI or to decrease infarct size at the time of reperfusion, as recently reported (17). However, the impact of prior beta blocker usage on immune cell function and responsiveness in human subjects is not well documented. We previously observed that beta blocker treatment of human macrophages increased VCAM-1 expression, similar to macrophages derived from $\beta_{2}$-AR-KO mice, and that VCAM-1 expression was increased in the spleens of human subjects treated chronically with beta blockers (14). These results revealed that prior beta blocker treatment can alter the molecular signature of leukocytes, potentially affecting their function and responsiveness to acute injury. Thus, in our current study, we used mouse models and leukocytes from patients to examine whether chronic beta blocker treatment alters leukocyte splenic localization, chemokine receptor expression, and migration to decrease leukocytes' responsiveness to acute injury in vivo. Our results demonstrate that chronic treatment with beta blockers decreases leukocyte responsiveness in both murine models and human peripheral blood leukocytes (PBLs), suggesting that beta blockers with $\beta_{2}$-AR selectivity are capable of fine-tuning innate immune responses to injury in vivo.

\section{Results}

Beta blocker infusion alters splenic leukocyte parameters over time in vivo. Our previous studies identified key molecular changes in leukocytes in response to hematopoietic cell-specific deletion of $\beta_{2}$-AR, including increased splenic VCAM-1 expression and leukocyte retention, as well as decreased leukocyte CCR2 expression in BM-derived leukocytes $(14,15)$. Therefore, we aimed to determine whether prior treatment with $\beta_{2}$-ARselective or clinically used beta blockers would similarly alter these leukocyte parameters. To this end, WT C57BL/6J mice were administered via osmotic minipump vehicle (Veh), ICI-118,551 (ICI, a $\beta_{2}$-AR-selective antagonist), carvedilol (Carv, a nonselective adrenergic receptor antagonist), a low dose of the $\beta_{1}$-AR-selective antagonist metoprolol (Met Low) — which has been shown to be selective for $\beta_{1}$-AR (18-20) and to block cardiac $\beta_{1}$-AR-mediated contractility (Supplemental Figure 1A; supplemental material available online with this article; https://doi.org/10.1172/jci.insight.99485DS1) — or a high, nonselective dose of metoprolol (Met High) $(21,22)$. Similar to changes in splenic parameters we reported in $\beta_{2}$-AR-KO bone marrow transplant (BMT) mice, infusion of mice with ICI resulted in a temporally dependent increase in spleen size and splenic VCAM-1 expression (Figure 1, A-D), which peaked after 2 weeks of infusion. Using this time point, the impact of metoprolol and carvedilol were also tested, revealing that both Met High and Carv increased spleen size and splenic VCAM-1 expression, while Met Low did not influence these parameters (Figure 1, E-H).

Previously, enhanced spleen size and VCAM-1 expression secondary to loss of leukocyte-expressed $\beta_{2}$-AR was shown to correlate with increased splenic retention of various leukocyte populations, including monocytes/macrophages, neutrophils, and mast cells (14). Thus, initially using immunohistochemical analysis we quantified these leukocyte populations within the splenic red pulp and white pulp (Supplemental Figure 1B) after beta blocker infusion. Similar to our data above, infusion with ICI enhanced the accumulation of monocyte/macrophage $\left(\mathrm{CD}^{+} 8^{+}\right)$lineage, mast cell (tryptase ${ }^{+}$) lineage, and neutrophil (myeloperoxidase, $\mathrm{MPO}^{+}$) lineage cells in a temporally dependent manner (Figure 2, A-D, and Supplemental Figure 2, A-D). Infusion with either Met High or Carv for 2 weeks also resulted in the splenic accumulation of CD68 ${ }^{+}$, tryptase ${ }^{+}$, and $\mathrm{MPO}^{+}$leukocytes (Figure 2, A-D), whereas Met Low had no significant impact on these parameters. In all cases, the changes in leukocyte parameters were observed in the red pulp, but not the white pulp, of the spleen (Supplemental Figure 2). In alignment with the immunohistochemistry results, flow cytometry analysis of cells isolated from the spleens of mice treated with ICI for 2 weeks also revealed enhanced accumulation of $\mathrm{CD}^{+} 5^{+} \mathrm{CD} 8^{+}$(hematopoiesis-derived monocyte/macrophage lineage), $\mathrm{CD} 45^{+} \mathrm{CD} 117^{+}$(hematopoiesis-derived mast cell lineage), and $\mathrm{CD} 45^{+} \mathrm{Ly}_{6 \mathrm{G}}{ }^{+}$(hematopoiesis-derived neutrophil lineage) leukocytes (Figure 3, A-F). These data support the concept wherein pharmacological inhibition of $\beta_{2}$-AR for as little as 2 weeks is sufficient to produce a detectable alteration in splenic leukocyte accumulation.

Chronic beta blocker treatment reduces CCR2 expression and migration of BM and PBLs. CCR2 mediates the recruitment of leukocytes to sites of injury in response to its ligand, CCL2 $(8,10,23,24)$. Because we had previously reported that complete loss of $\beta_{2}$-AR expression in leukocytes resulted in a marked reduction in CCR2 expression and BMC responsiveness to CCL2-mediated migration (15), we tested whether beta blocker infu- 
A

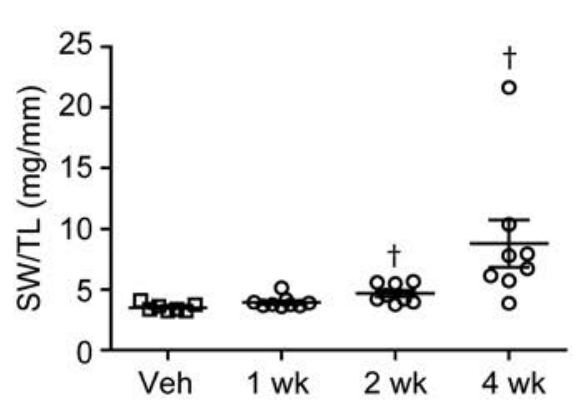

B

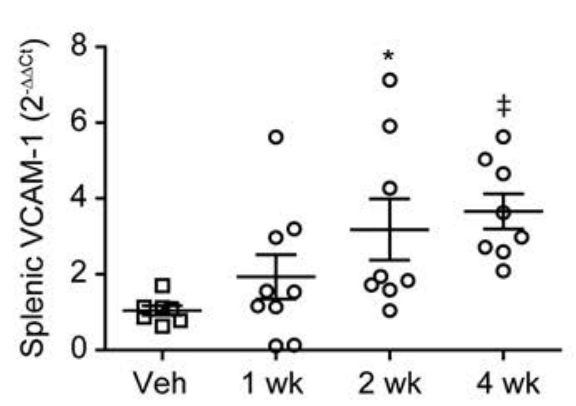

D

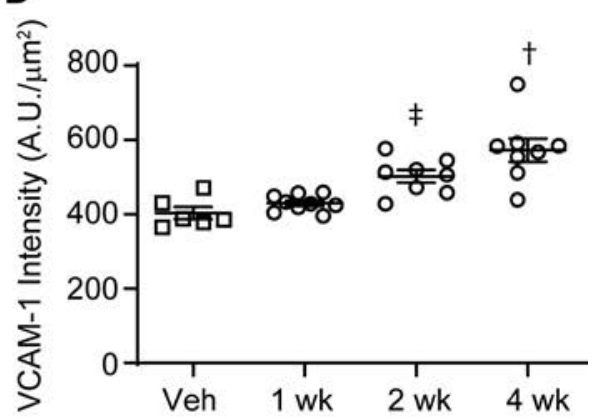

C

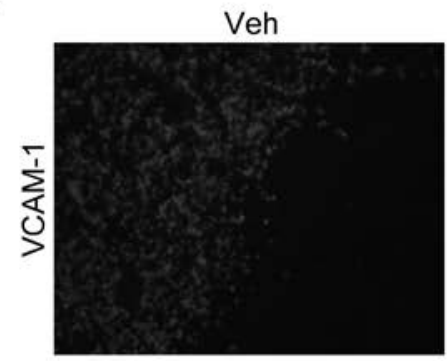

E

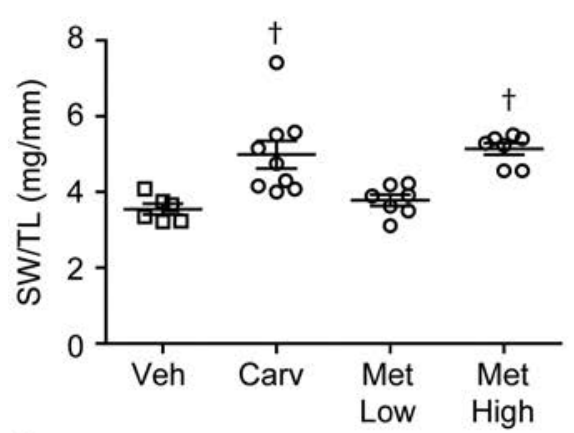

$1 \mathrm{wk}$

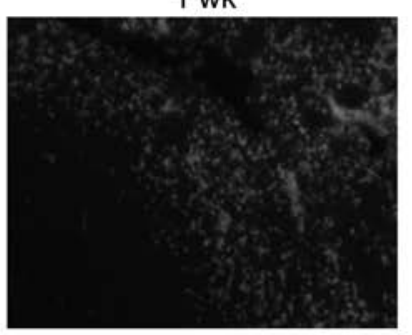

$\mathbf{F}$

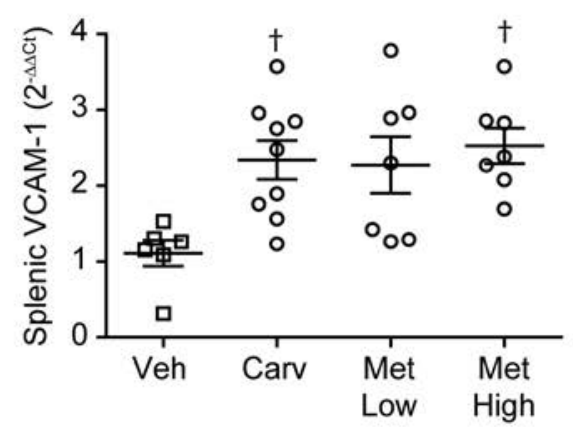

2 wk

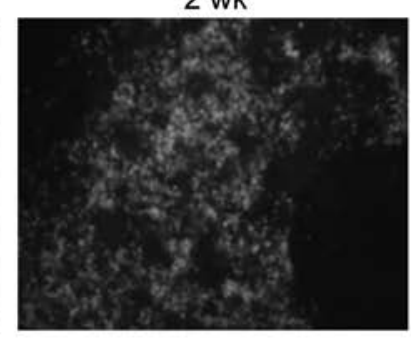

4 wk

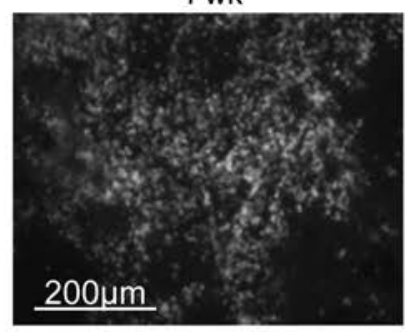

G
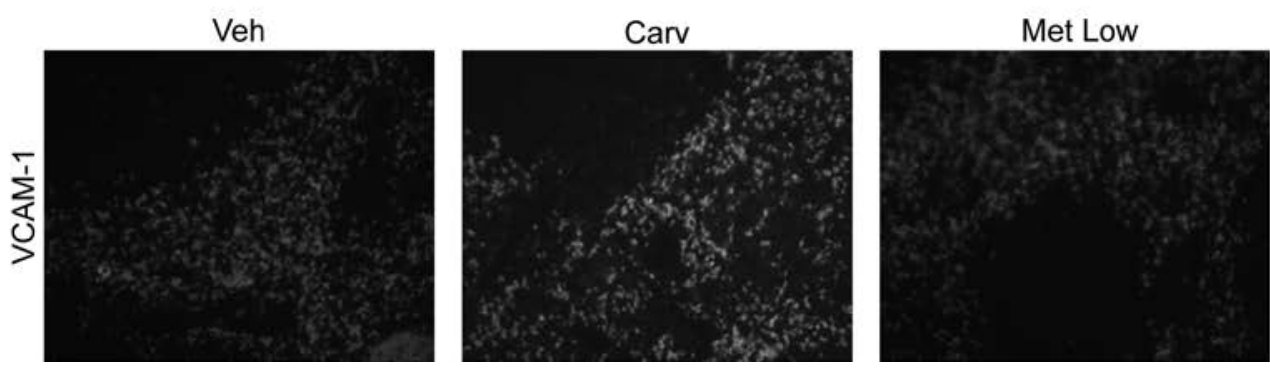

H
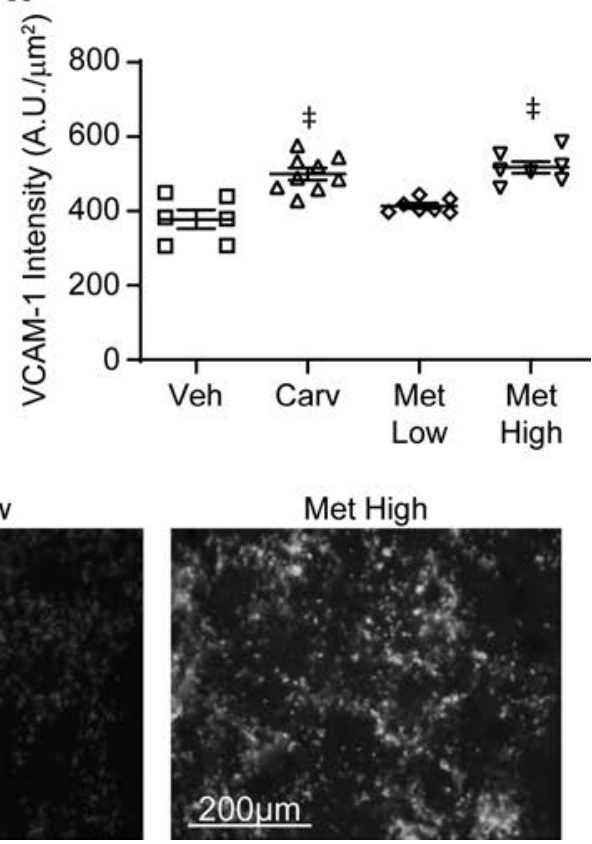

Figure 1. Beta blocker infusion alters baseline splenic parameters. (A and E) Spleen size was quantified by gravimetric analysis (SW) and normalized to tibia length (TL). (B and F) Reverse transcription quantitative PCR (RT-qPCR) was used to measure splenic VCAM-1 expression. (C, D, G, and $\mathbf{H})$ Immunohistochemistry was used to detect splenic VCAM-1 expression from mice infused with (A-D) Veh or ICl for 1 to 4 weeks or (E-F) Carv, Met Low, or Met High for 2 weeks via osmotic minipumps. Overall Kruskal-Wallis test $P$ values: $(\mathbf{A}) P=0.0001,(\mathbf{B}) P=0.0031,(\mathbf{D}) P=0.0008,(\mathbf{E}) P=0.0004,(\mathbf{F}) P=0.0116$, and $(\mathbf{H})$ $P<0.0001$. (A and B) $n=7$ for Veh, $n=9$ for 1-week ICI, $n=8$ for 2-week ICI, and $n=8$ for 4-week ICI. (D) $n=6$ for Veh, $n=9$ for 1-week ICI, $n=8$ for 2-week ICI, and $n=8$ for 4-week ICI. (E, F, and $\mathbf{H}) n=6$ for Veh, $n=9$ for Carv, $n=7$ for Met Low, and $n=7$ for Met High. Results of exact Wilcoxon rank-sum tests with multiple-comparison adjustment - 3 comparisons, (A-D) time points versus Veh or (E-H) beta blocker versus Veh - are indicated in scatter dot plots. ${ }^{*} P<0.05,{ }^{\dagger} P<0.01$, and ${ }^{\ddagger} P<0.001$ versus Veh. Scale bar: $200 \mu \mathrm{m}$.

sion is also sufficient to alter these parameters. Indeed, infusion with ICI reduced BMC-expressed CCR2 in a temporally dependent manner (Figure 4A), while infusion with Carv or Met High for 2 weeks also resulted in decreased BMC CCR2 expression, and Met Low had no impact (Figure 4B). Via flow cytometry, decreased levels of $\mathrm{CD} 45^{+} \mathrm{CCR} 2^{+}$(hematopoiesis-derived CCR2 $2^{+}$cells) in BMCs following 2 weeks of ICI infusion were also confirmed (Figure 4, C and D). Molecular changes within the BM do not necessarily represent those that 


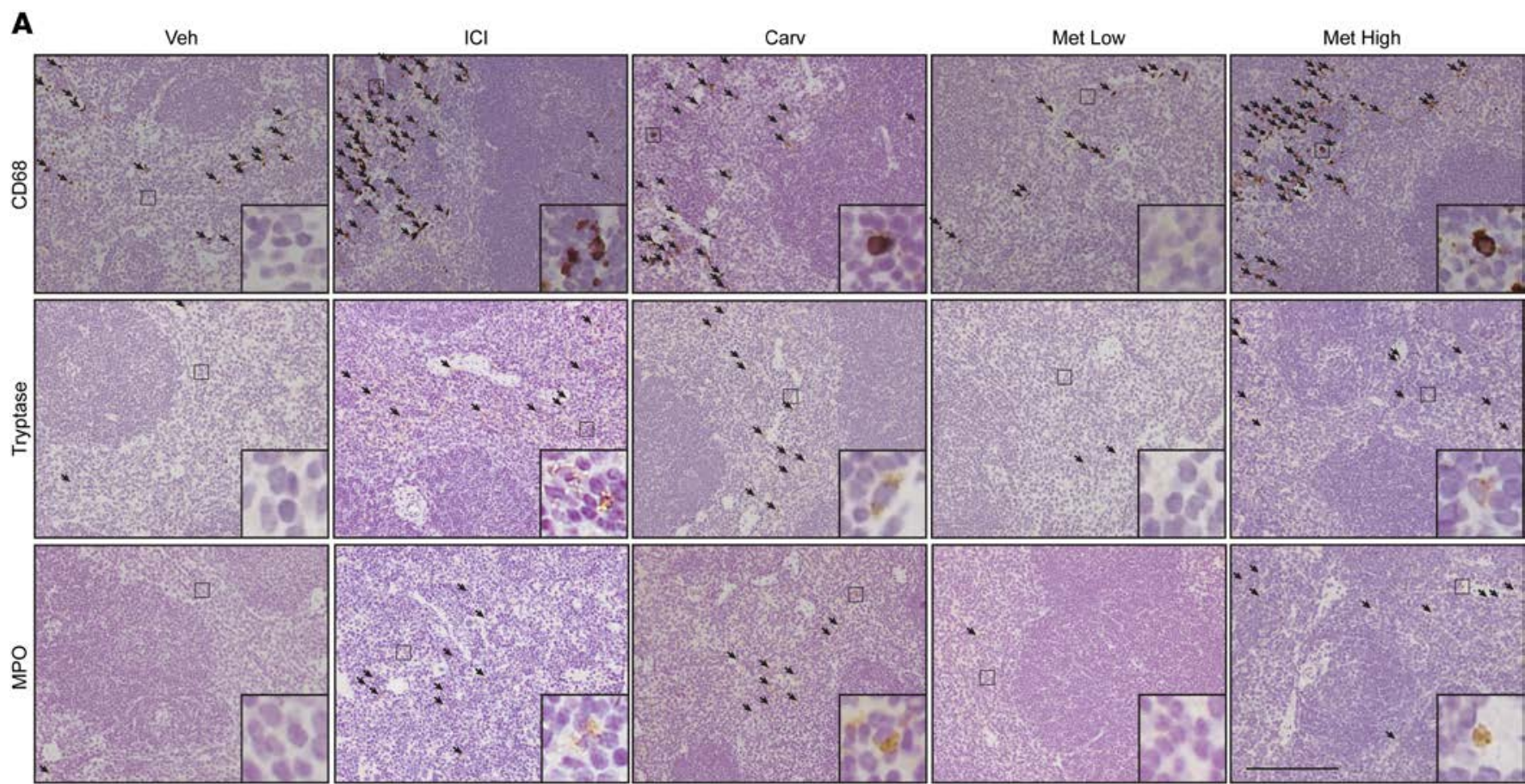

B

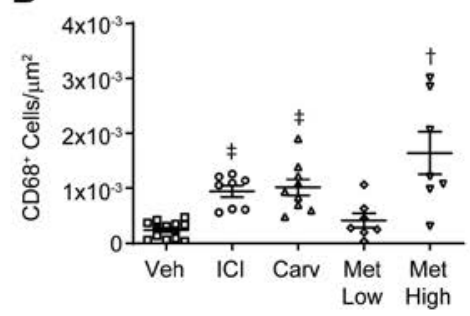

C

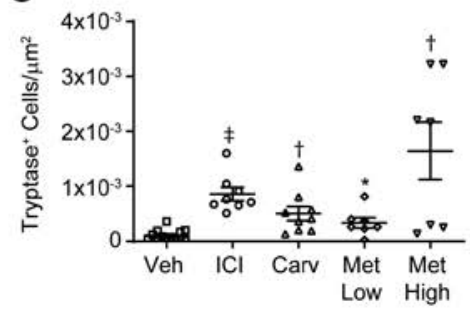

D

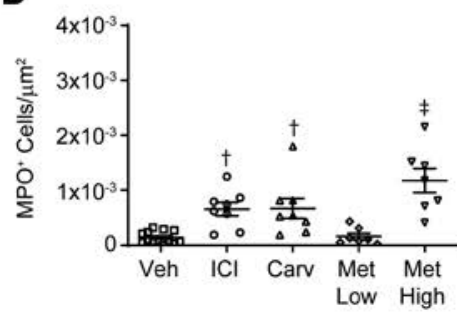

Figure 2. Beta blocker infusion alters baseline splenic leukocyte localization. (A) Representative CD68, tryptase, and MPO staining (black arrowheads) within the spleens of mice that were administered Veh, ICI, Carv, Met Low, or Met High for 2 weeks. Scale bar: $200 \mu \mathrm{m}$. Quantification of (B) CD68+, (C) tryptase ${ }^{+}$, and (D) $\mathrm{MPO}^{+}$cells within the splenic red pulp from A. Overall Kruskal-Wallis test $P$ values: (B) $P<0.0001,(\mathbf{C}) P=0.0001$, and (D) $P<0.0001$. $n=13$ for Veh, $n=8$ for ICI, $n=9$ for Carv, $n=7$ for Met Low, and $n=7$ for Met High. Results of exact Wilcoxon rank-sum tests with multiple-comparison adjustment -4 comparisons, beta blockers versus Veh - are indicated in scatter dot plots. ${ }^{\dagger} P<0.01$, and ${ }^{\ddagger} P<0.001$ versus Veh.

may occur in circulating leukocytes; thus, we sought to determine whether the same responses to beta blockers we observed in isolated BMCs could also be detected in PBLs. To this end, we isolated buffy coats containing the PBL population from mice treated with each beta blocker for 2 weeks and measured CCR2 expression. Similar to our observations in BMCs, CCR2 expression was significantly decreased in PBLs chronically exposed to ICI, Carv, and Met High but not Met Low (Figure 4E). Because CCR2 expression was reduced in both BMCs and PBLs by $\beta_{2}$-AR-selective beta blockade, we tested whether these cells exhibited impaired migration toward CCL2. Responsiveness to CCL2 was almost completely abrogated in both BMCs and PBLs by prior infusion with ICI, Carv, or Met High (Figure 5, A-C). In contrast, CCL2-mediated migration was unaffected in either leukocyte population obtained from Met Low-infused mice.

Although mice provide a useful in vivo model to explore the effects of beta blocker treatment on leukocytes, we aimed to determine whether our results translate to human leukocyte biology. Therefore, to determine whether a molecular readout of beta blocker-mediated changes in leukocytes can be also detected in human PBLs, we analyzed CCR2 expression and responsiveness in buffy coat samples obtained from patients treated chronically with or without beta blockers. Similar to our data obtained from mice, CCR2 expression was significantly reduced in the PBLs of patients who had been receiving chronic beta blocker therapy compared with beta blocker-naive patients (Table 1). Further, PBLs of patients having received chronic beta blocker treatment displayed reduced migratory responsiveness to CCL2 (Figure 5, D and E). In all, these data highlight the translatability of our mouse model data to clinical relevance in humans. 
A

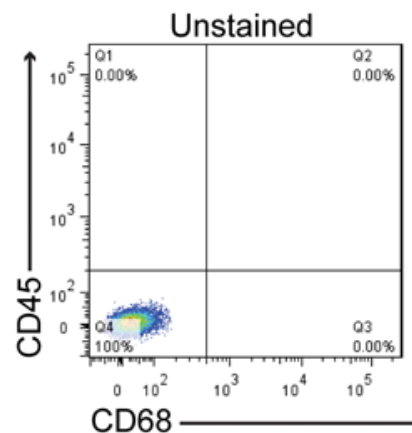

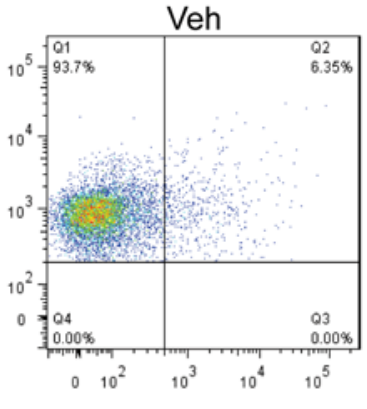

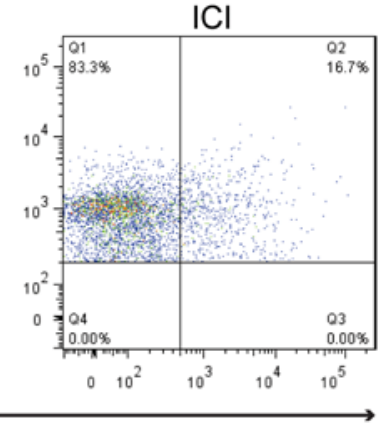

C
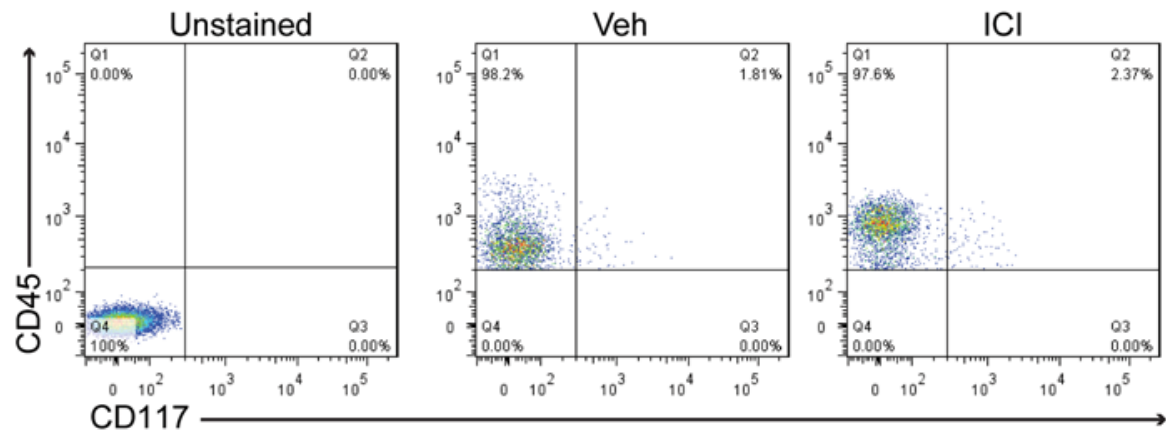

E
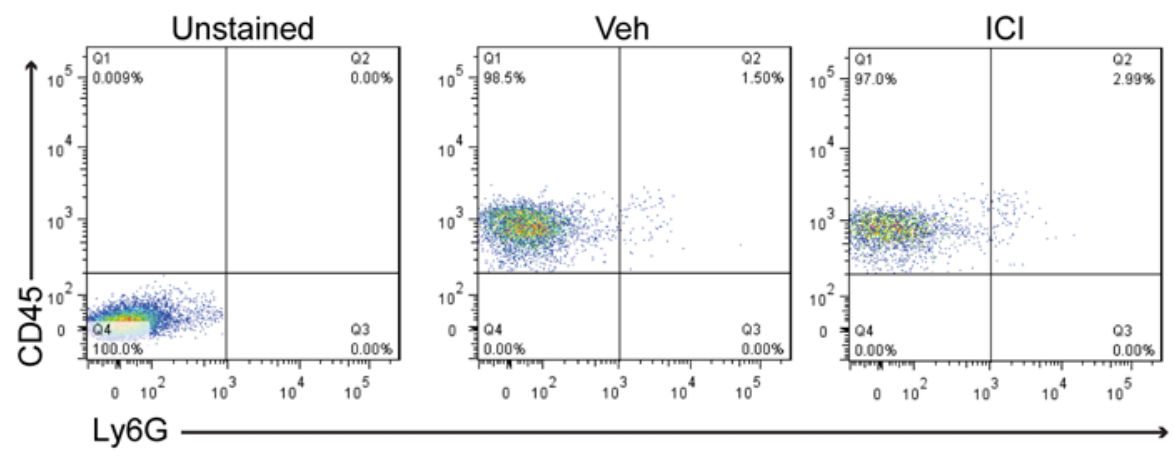

B

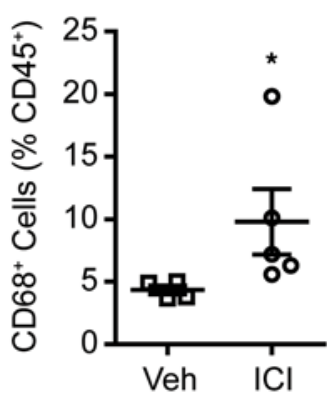

D

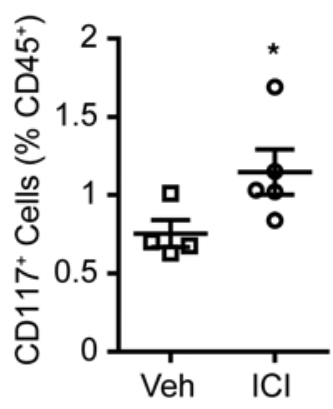

$\mathbf{F}$

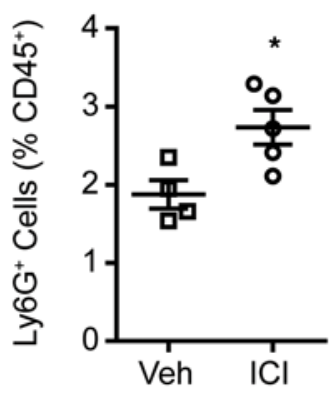

Figure 3. Flow cytometry analysis of splenic leukocyte accumulation following infusion of ICI-118,551. Via flow cytometry, (A and B) CD45+CD68 ${ }^{+}$, (C and D) $\mathrm{CD}_{45}{ }^{+} \mathrm{CD} 117^{+}$, and (E and F) CD45+Ly6C+ leukocytes were quantified from the spleens of mice infused for 2 weeks with Veh or ICI (unstained controls at left; isotype controls are shown in Supplemental Figure 7). $n=4$ Veh, and $n=5 \mathrm{ICI} .{ }^{*} P<0.05$, exact Wilcoxon rank-sum test.

Prior beta blocker infusion reduces leukocyte responsiveness to injury in vivo. We previously reported that mice with $\beta_{2}$-AR deletion specifically in cells of hematopoietic origin displayed reduced infiltration of leukocytes into the heart following acute MI $(14,15)$. Because chronic infusion with beta blockers induced similar baseline changes in both splenic and BM leukocytes, as observed in $\beta_{2}$-AR-KO BMT mice, populations that are integral to innate immune responses after injury $(2,5,6,25)$, we sought to determine whether chronic beta blocker treatment influences leukocyte responsiveness to MI-induced injury. Therefore, mice were administered Veh, ICI, Carv, Met High, or Met Low for 2 weeks, after which the pumps were removed to allow drug washout for 1 day before sham or MI surgery. Infarct length, as assessed at both 4 and 21 days after MI, was not different between Veh and any of the beta blocker treatment groups (Figure 6, A-C). Via echocardiography, we also tracked the cardiac function of a cohort of mice infused with beta blockers before sham or MI surgery for up to 3 weeks after MI. Although all mice receiving MI experienced a significant reduction in contractility compared with sham-operated mice as estimated by ejection fraction (Supplemental Figure 3A), there were no significant post-MI differences between any of the beta blocker pretreatment groups. However, prior treatment with ICI, Carv, or Met High did significantly reduce survival by approximately $50 \%$ to $60 \%$ within the first week because of increased cardiac rupture events (Figure 6D) such that there were limited mice remaining in some groups with which to assess cardiac function. Met Low did not significantly reduce survival when compared with Veh-infused mice. 
A

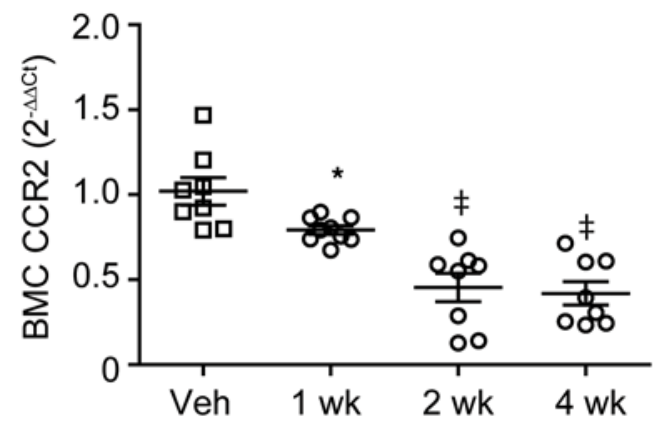

B

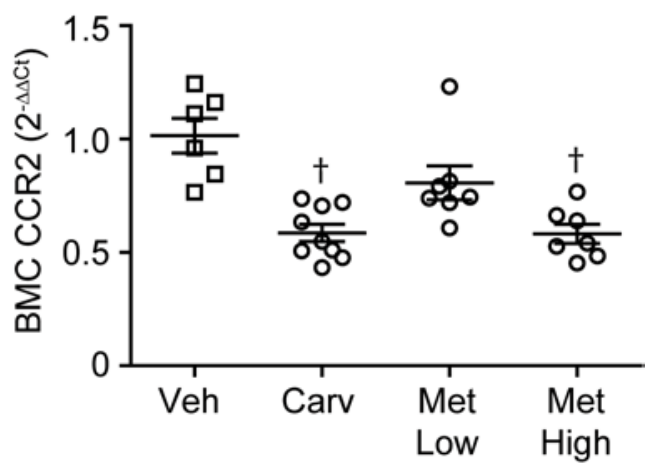

C
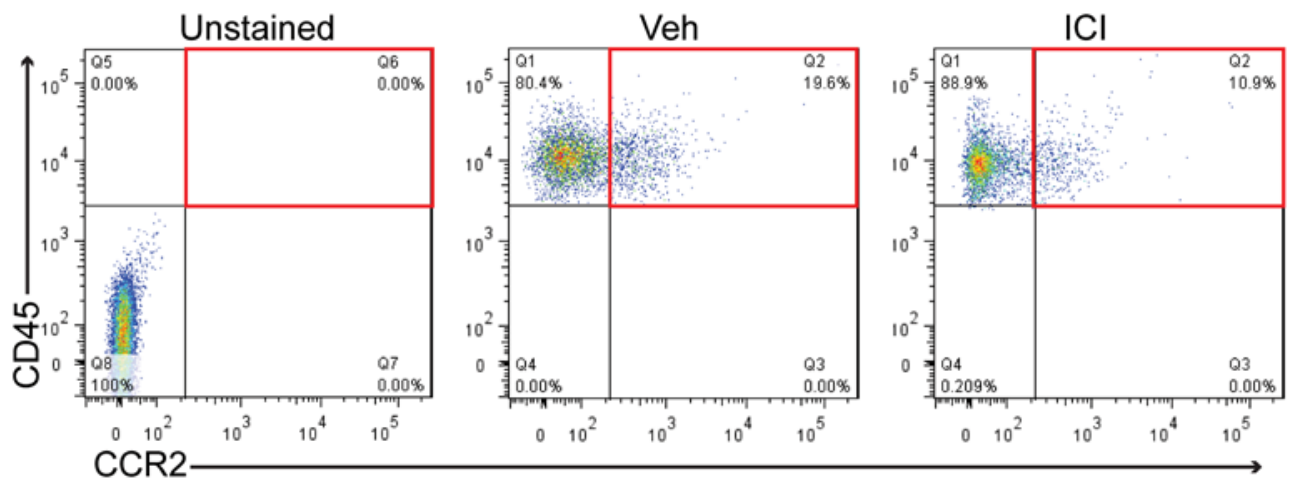

D

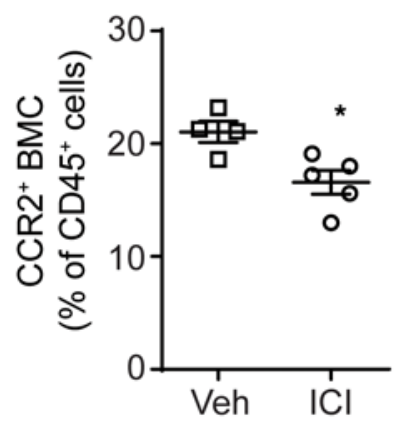

E

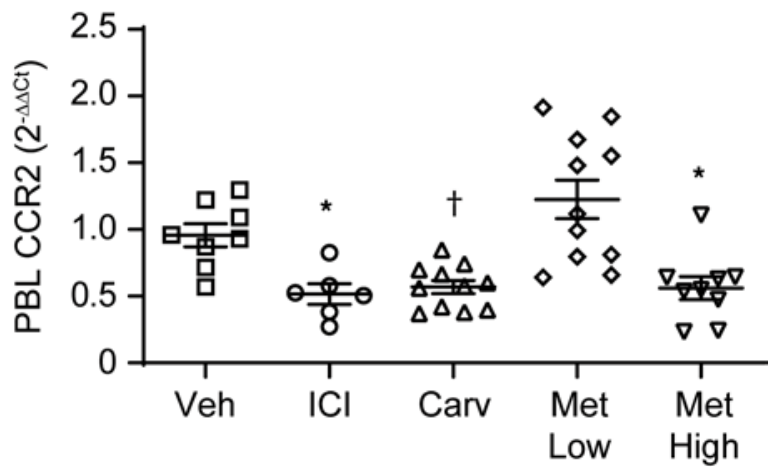

Figure 4. Beta blocker infusion decreases CCR2 expression in BMCs and PBLs. RT-qPCR was used to measure BMC CCR2 expression of WT C57BL/6) mice treated with (A) Veh versus ICI for increasing time points or (B) Veh versus Carv, Met Low, or Met High for 2 weeks via osmotic minipumps. Overall Kruskal-Wallis test $P$ values: (A) $P<0.0001$, and (B) $P=0.0006$. (A) $n=8$ for Veh, $n=9$ for 1 week, $n=8$ for 2 weeks, and $n=8$ for 4 weeks. (B) $n=6$ for Veh, $n$ $=9$ for Carv, $n=7$ for Met Low, and $n=7$ for Met High. Results of exact Wilcoxon rank-sum tests with multiple-comparison adjustment -3 comparisons, (A) time points versus Veh or (B) beta blocker versus Veh - are indicated in scatter dot plots. ${ }^{*} P<0.05,{ }^{\dagger} P<0.01$, and ${ }^{\ddagger} P<0.001$ versus Veh. (C and D) Via flow cytometry, $C D 45^{+} C C R 2^{+} B M C s$ were quantified from mice infused for 2 weeks with Veh or ICI (unstained controls at left; isotype controls are shown in Supplemental Figure 7). $n=4$ Veh, and $n=5 \mathrm{ICI}$. ${ }^{*} P<0.05$, exact Wilcoxon rank-sum test. (E) RT-qPCR was used to measure PBL CCR2 expression from WT C57BL/6] mice treated with Veh versus ICI, Carv, Met Low, or Met High for 2 weeks via osmotic minipumps. Overall Kruskal-Wallis test $P$ value: $P=0.0001$. $n=8$ for Veh, $n=6$ for ICI, $n=11$ for Carv, $n=12$ for Met Low, and $n=9$ for Met High. Results of exact Wilcoxon rank-sum tests with multiple-comparison adjustment -4 comparisons, beta blocker versus Veh - are indicated in scatter dot plots. ${ }^{*} P<0.05$, and ${ }^{\dagger} P<0.01$ versus Veh.

Because $\beta_{2}$-AR-KO BMT mice experienced $100 \%$ mortality following MI (14), but beta blockerinfused mice displayed variable mortality outcomes, these data suggest an intermediate effect of prior beta blocker treatment on leukocyte parameters. As such, we assessed splenic VCAM-1 and leukocyte levels, BMC CCR2 expression, and cardiac leukocyte infiltration at 4 days after MI. Although splenic VCAM-1 expression trended toward increased by ICI versus Veh in both sham-treated and MI mice 
A
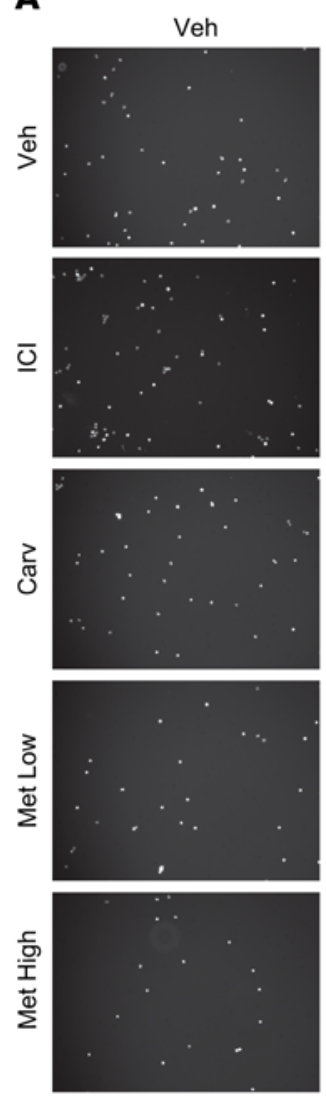

D

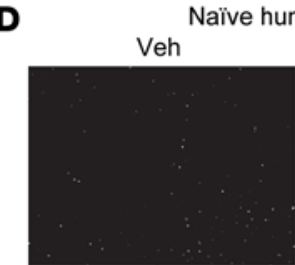

BMC
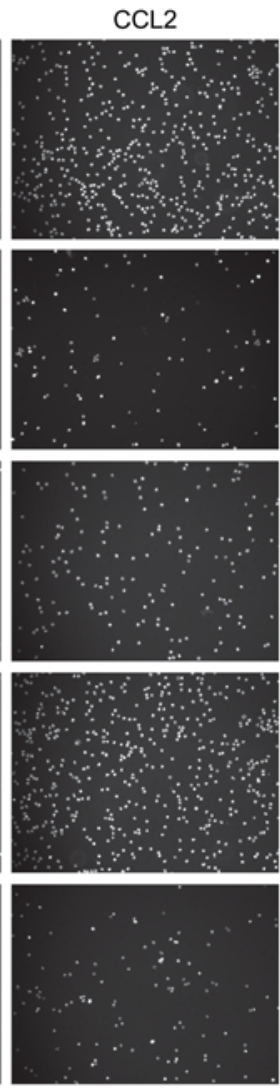

CCL2

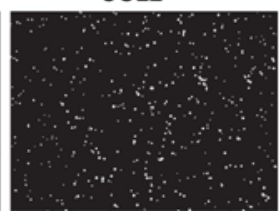

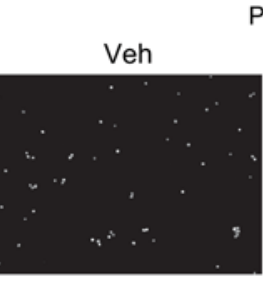
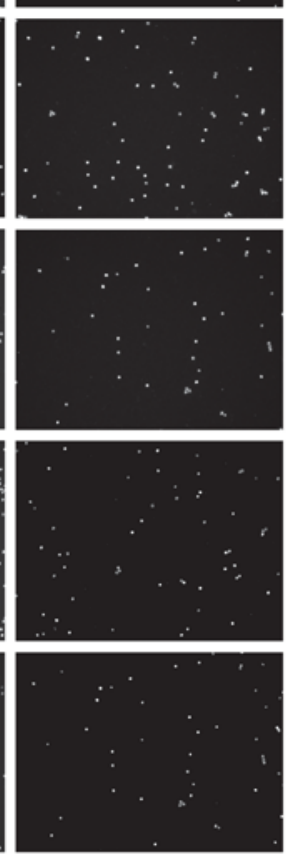

$\beta$

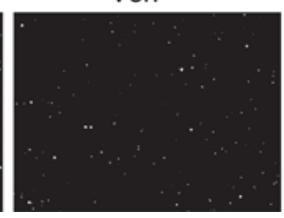

BL
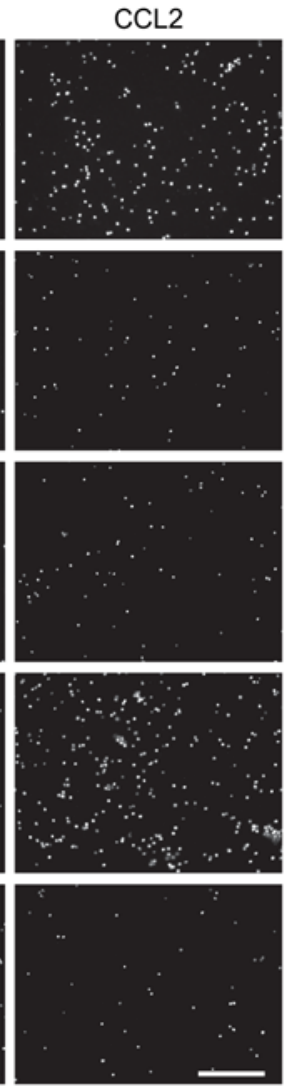

B

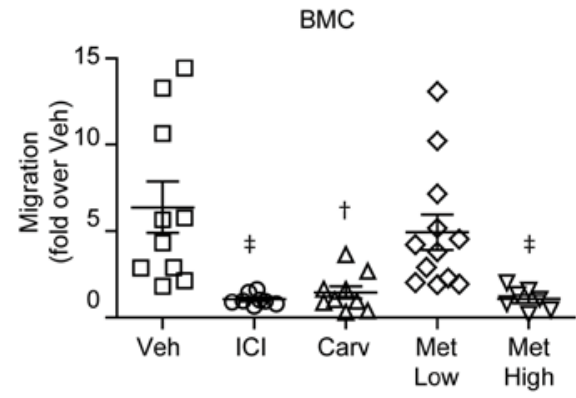

C

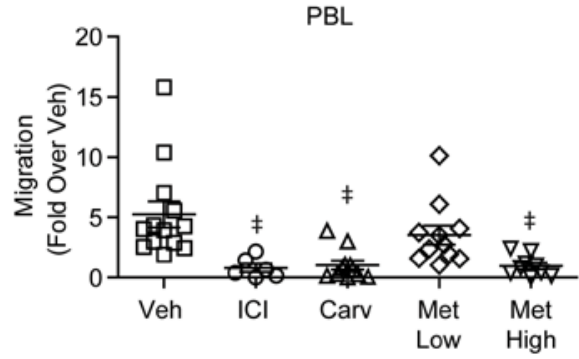

E

Human PBL

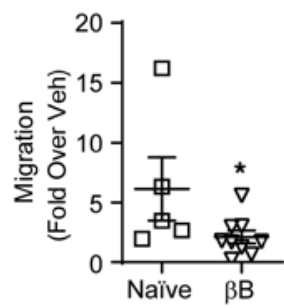

Figure 5. Prior beta blocker infusion decreases mouse and human leukocyte migration. (A) Representative Hoechst staining from Transwell migration assays of BMCs or PBLs isolated from WT mice infused for 2 weeks with Veh, ICI, Carv, Met Low, or Met High and treated with Veh or CCL2 for 4 hours. Scale bar: $200 \mu \mathrm{m}$. Quantification of migration assay results for (B) BMCs or (C) PBLs. Values are expressed as fold change over Veh-stimulated migration. Overall Kruskal-Wallis test $P$ values: (B) $P<0.0001$, and (C) $P=0.0001$. (B) $n=10$ for Veh, $n=8$ for ICl, $n=9$ for Carv, $n=12$ for Met Low, and $n=7$ for Met High. (C) $n=13$ for Veh, $n=6$ for ICl, $n=11$ for Carv, $n=12$ for Met Low, and $n=9$ for Met High. Results of exact Wilcoxon rank-sum tests with multiple comparison adjustment -4 comparisons, beta blockers versus Veh - are indicated in scatter dot plots. ${ }^{\dagger} P<0.01$, and ${ }^{\ddagger} P<0.001$ versus Veh. (D) Representative Hoechst staining from Transwell migration assays of human PBL-isolated patients taking beta blockers or beta blocker-naive patients who were treated with Veh or CCL2 for 4 hours. Scale bar: $200 \mu \mathrm{m}$. (E) Quantification of migration assay results from $\mathbf{D}$, where values are expressed as fold change over Veh-stimulated migration. $n=5$ beta blocker-naive patients, and $n=9$ beta blocker-treated patients. ${ }^{*} P<0.05$, exact Wilcoxon rank-sum test.

(Supplemental Figure 3B), spleen weights after procedures (Supplemental Figure 3C) were not significantly altered by any of the beta blocker treatment regimens versus Veh-treated mice ( 5 days following beta blocker cessation). Conversely, mice treated with ICI, Carv, or Met High displayed decreased expression of CCR2 in their BMCs, which was more apparent 4 days after MI (Figure 6E), while Met Low had no effect. Correspondingly, cardiac infiltration of $\mathrm{CD}^{+} 8^{+}$, tryptase ${ }^{+}$, and $\mathrm{MPO}^{+}$cells into the border zone of hearts after MI was each significantly decreased in mice that had received prior treatment with ICI, Carv, or Met High compared with those that received Veh, while leukocyte infiltration was unaffected by prior treatment with Met Low (Figure 7, A-D). None of the beta blocker regimens altered leukocyte numbers in sham-operated mouse hearts or the remote zone of hearts from MI mice (Supplemental Figure 4, A-G). Flow cytometry analysis of cardiac nonmyocytes isolated 4 days after MI from mice pretreated for 2 weeks with Veh or ICI before MI surgery showed significantly decreased 
Table 1. Patient demographics and CCR2 expression in human PBLs

\begin{tabular}{|c|c|c|}
\hline & Beta blocker naive $(n=27)$ & Beta blocker treated $(n=38)$ \\
\hline Mean age in years \pm SEM (range) & $56.3 \pm 2.9(24-77)$ & $58.5 \pm 2.0(25-80)$ \\
\hline Mean weight in kg \pm SEM (range) & $80.2 \pm 3.5(41-109)$ & $88.0 \pm 3.6(53-138)$ \\
\hline \multicolumn{3}{|l|}{ Sex } \\
\hline Male (\%) & $11(40.7)$ & $26(68.4)$ \\
\hline Female (\%) & $15(55.6)$ & 12 (31.6) \\
\hline Not reported (\%) & $1(3.7)$ & $0(0)$ \\
\hline \multicolumn{3}{|l|}{ Ethnicity } \\
\hline White (\%) & $11(40.7)$ & $20(52.6)$ \\
\hline Black or African American (\%) & $6(22.2)$ & $11(28.9)$ \\
\hline Not reported (\%) & $10(37.0)$ & $7(18.4)$ \\
\hline \multicolumn{3}{|l|}{ Cardiovascular Disease Status } \\
\hline HTN (\% within treatment group) & $12(44.4)$ & $22(57.9)$ \\
\hline DM (\% within treatment group) & $6(22.2)$ & $14(36.8)$ \\
\hline CAD (\% within treatment group) & $8(29.6)$ & $11(28.9)$ \\
\hline HF (\% within treatment group) & $10(37.0)$ & $21(55.3)$ \\
\hline \multicolumn{3}{|l|}{ NYHA Class for HF Patients } \\
\hline 1 (\% within treatment group) & $1(10)$ & $1(4.76)$ \\
\hline 2 to 3 (\% within treatment group) & $2(20)$ & $13(61.9)$ \\
\hline 3 to 4 (\% within treatment group) & $7(70)$ & $6(28.6)$ \\
\hline CCR2 expression as RQ relative to control & 1.01 & $0.44^{\mathrm{A}}$ \\
\hline (range indicated by $\mathrm{RQ}_{\min }$ and $\left.\mathrm{RQ}{ }_{\max }\right)^{\text {) }}$ & $(0.85-1.17)$ & $(0.33-0.55)$ \\
\hline \multicolumn{3}{|c|}{ HTN, hypertension; DM, diabetes mellitus; CAD, coronary artery disease; HF, heart failure; NYHA, New York Heart } \\
\hline \multicolumn{3}{|c|}{$\begin{array}{l}\text { Association; CCR2, chemokine receptor } 2 \text {; RQ, relative quantitation. }{ }^{A} P<0.05 \text { versus beta blocker-naive group, exact } \\
\text { Wilcoxon rank-sum test. }\end{array}$} \\
\hline
\end{tabular}

accumulation of $\mathrm{CD} 45^{+} \mathrm{CD} 68^{+}$cells and a trend toward decreased $\mathrm{CD} 45^{+} \mathrm{Ly}_{6 \mathrm{G}}{ }^{+}$cells within the heart 4 days after MI, although no difference was observed for $\mathrm{CD} 45^{+} \mathrm{CD} 117^{+}$cells (Supplemental Figure 5 , A-F). These results suggest that despite recovery of splenic leukocyte parameters by 5 days after MI, beta blocker-mediated changes in leukocyte CCR2 expression and responsiveness are more long-lasting, thereby diminishing leukocyte infiltration into the heart after acute cardiac injury. Altogether, our study highlights the ability of chronic beta blocker treatment to alter baseline leukocyte characteristics that decrease leukocytes' responsiveness to acute injury and suggests that prior beta blockade may act, if properly titrated, to reduce the severity of immune responses to injury.

\section{Discussion}

We previously demonstrated that WT mice having received transplantation of $\beta_{2}-\mathrm{AR}-\mathrm{KO} \mathrm{BM}$, in which $\beta_{2}$-AR signaling was completely absent in leukocytes, were unable to achieve leukocyte egress from the spleen and recruitment to the heart following MI $(14,15)$. These data suggested that beta blockers, used extensively in the treatment of numerous clinical etiologies, could act to alter the molecular characteristics of leukocytes at baseline and reduce their responsiveness to acute injury. Therefore, in this study, we examined the impact of prior treatment with both $\beta_{2}$-AR-selective and clinically used beta blockers on several leukocyte parameters, including splenic localization, CCR2-mediated migration, and recruitment to the heart following injury. Indeed, we demonstrate that chronic treatment with $\beta_{2}$-AR-selective and nonselective beta blockers altered baseline splenic expression of VCAM-1 and leukocyte localization, as well as leukocyte-specific CCR2 expression and responsiveness to CCL2-mediated migration. Further, mice that underwent prior treatment with $\beta_{2}$-AR-selective and nonselective beta blockers displayed markedly impaired recruitment of leukocytes to the injured heart. Although in accordance with our previous studies $(14,15)$, this study specifically highlights several important facets of the impact of prior beta blocker treatment on leukocyte responsiveness to injury. 

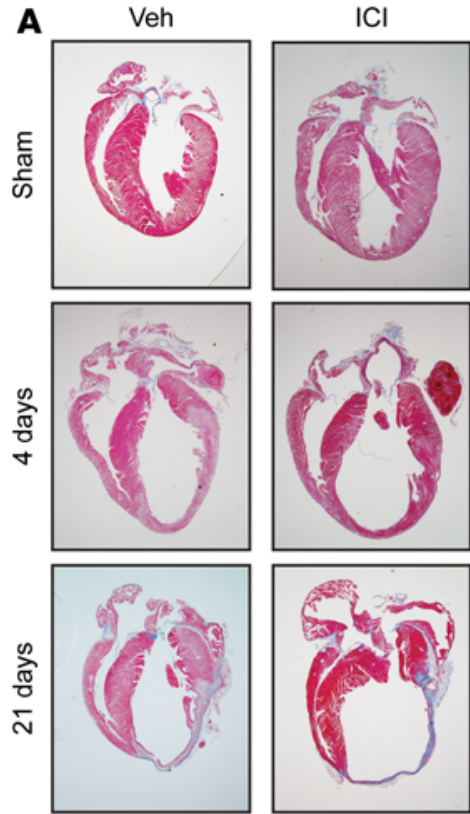

D

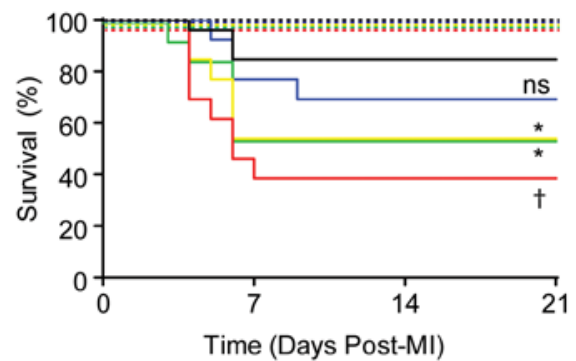

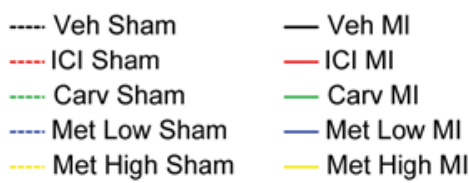
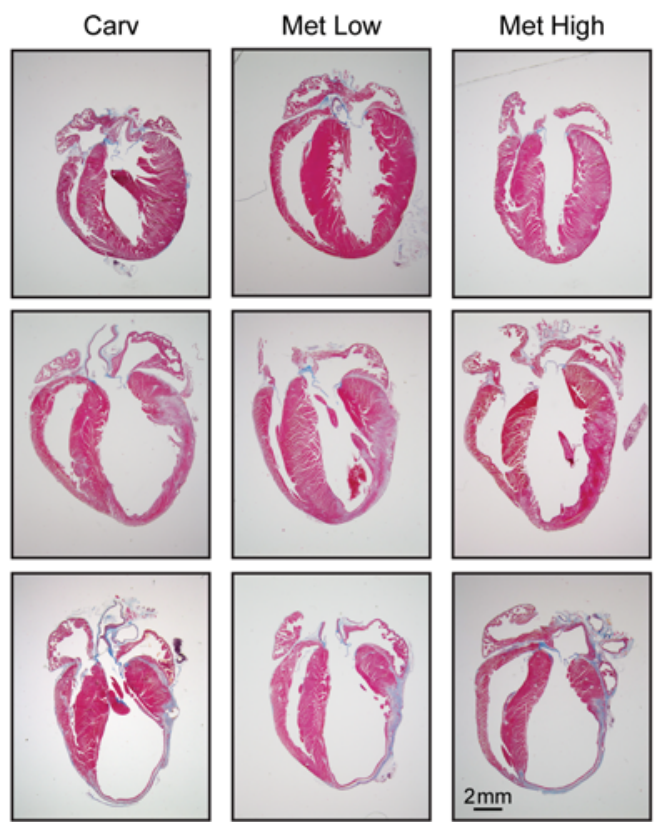

E
B

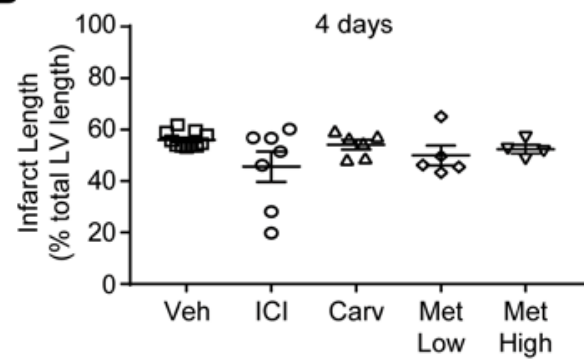

C

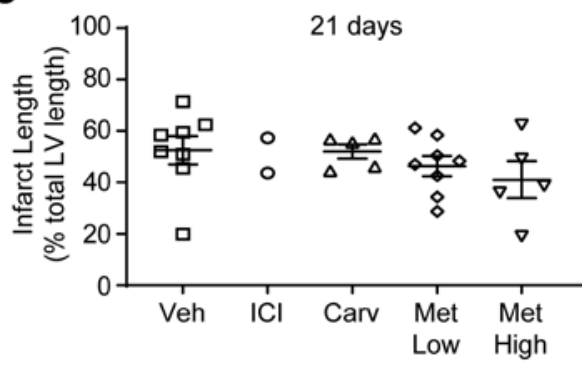

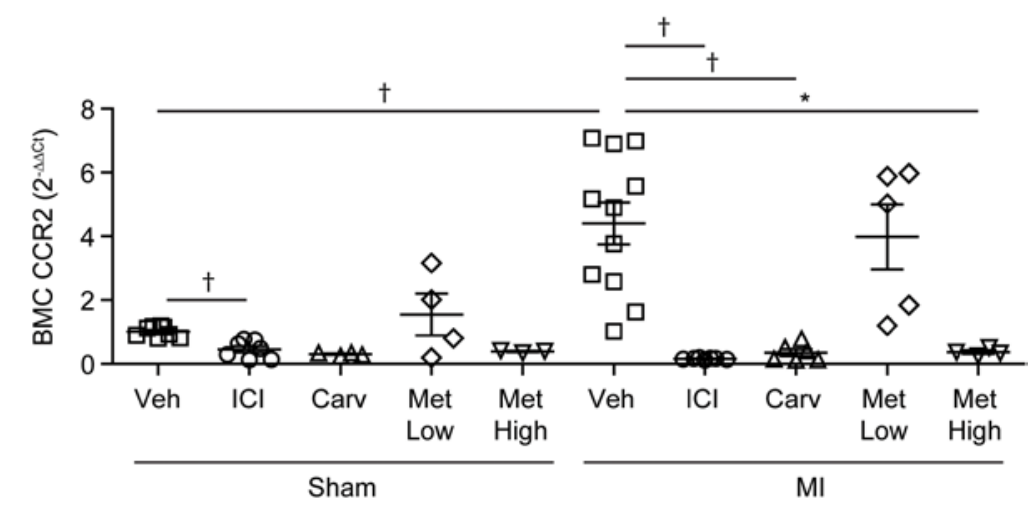

Figure 6. Chronic beta blocker infusion before MI does not affect infarct size but does alter survival and CCR2 expression. (A) Representative Masson's trichrome staining of infarcted hearts, 4 and 21 days after MI, from mice treated for 2 weeks with Veh, ICI, Carv, Met Low, or Met High before surgery. For 4 days: Veh $(n=11)$, ICI $(n=7)$, Carv $(n=6)$, Met Low $(n=5)$, or Met High $(n=4)$; for 21 days: Veh $(n=8)$, ICI $(n=2)$, Carv $(n=5)$, Met Low $(n=8)$, or Met High $(n=5)$. Scale bar: $2 \mathrm{~mm}$. (B and C) Quantification of infarct lengths from A. (D) Survival of mice that had received infusion of Veh, ICI, Carv, Met Low, or Met High for 2 weeks before sham or MI surgery, as monitored daily for 21 days after MI. Log-rank test: ${ }^{*} P<0.05$, and ${ }^{\dagger} P<0.01$ versus Veh MI. Starting $n=10$ for Veh-treated sham group; $n=5$ for ICI sham; $n=4$ for Carv sham, Met High sham, and Met Low sham; $n=26$ for Veh MI; and $n=13$ for ICI MI, Carv MI, Met High MI, and Met Low MI. (E) RT-pPCR was used to quantify BMC CCR2 expression from sham-treated and 4-day post-MI mice that were administered Veh, ICI, Carv, Met Low, or Met High for 2 weeks before surgery. Overall Kruskal-Wallis test $P$ value: $P<0.0001$. $n=8$ for Veh sham, $n=11$ for Veh MI, $n=7$ for ICI sham, $n=7$ for ICI MI, $n=4$ for Carv sham, $n=6$ for Carv MI, $n=4$ for Met Low sham, $n=5$ for Met Low MI, $n=3$ for Met High sham, and $n=4$ for Met High MI. Results of exact Wilcoxon rank-sum tests with multiple-comparison adjustment -13 comparisons: sham beta blockers versus MI beta blockers, sham beta blockers versus sham Veh, and MI beta blockers versus MI Veh - are indicated in scatter dot plots. ${ }^{*} P<0.05$, and ${ }^{\dagger} P<0.01$.

First, $\beta_{2}$-AR selectivity is critical in the ability of a beta blocker to impart effects on splenic accumulation and leukocyte recruitment to the site of injury. Pretreatment of mice with ICI-118,551 (a strong inverse $\beta_{2}$-AR agonist, ref. 26), carvedilol (a nonselective $\beta$-AR antagonist), and a high, nonselective dose of the neutral $\beta_{1}$-AR antagonist metoprolol (21) each increased splenic VCAM-1 expression and leukocyte accumulation while reducing leukocyte CCR2 expression and responsiveness to injury as well as survival after MI. Most compellingly, metoprolol did not modulate leukocyte molecular parameters or responsiveness when infused at a low, $\beta_{1}$-AR-selective dose, which still blocked catecholamine-induced cardiac contractility. These results suggest that both carvedilol and metoprolol, when used at a nonselective dose, allow $\beta_{2}$-AR antagonism that can alter the molecular blueprint of leukocytes and their resulting ability to respond to acute injury. However, via allometric scaling from a human average weight of approximately $85 \mathrm{~kg}$ in 
A
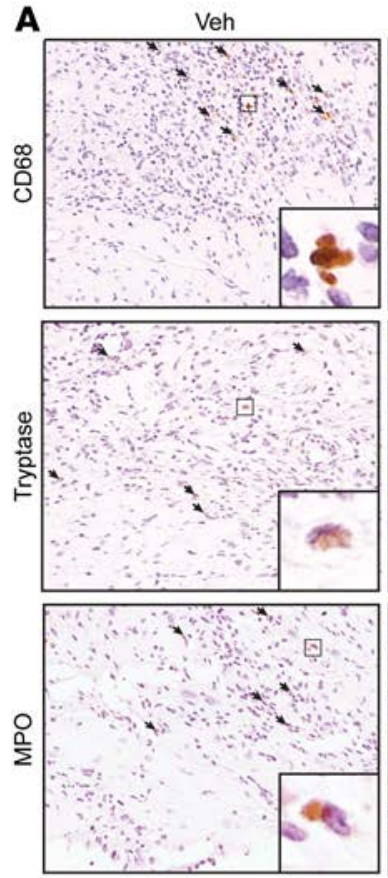

B

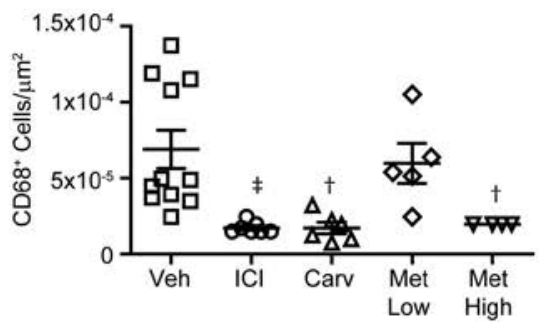

$\mathrm{ICl}$
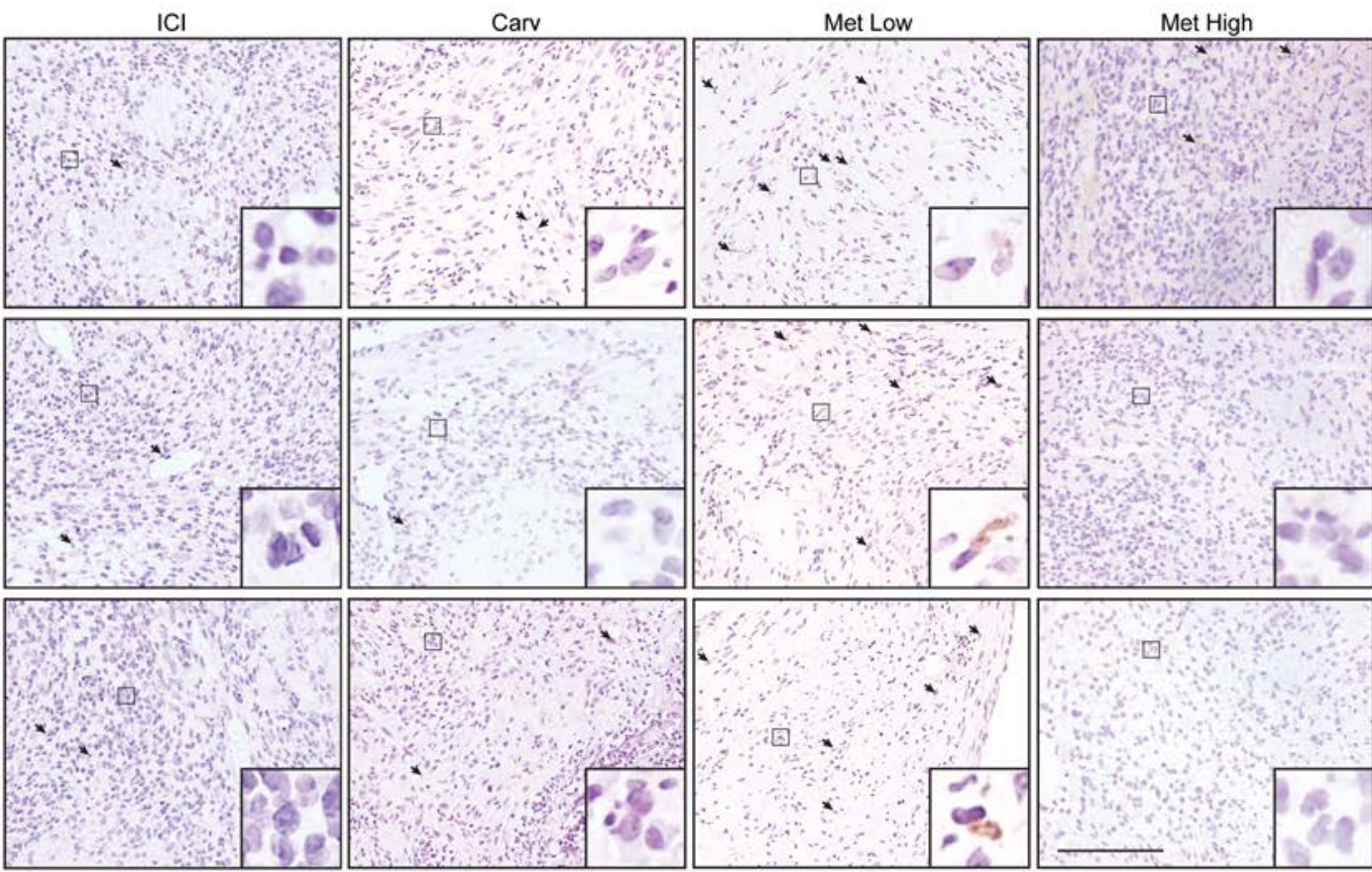

C

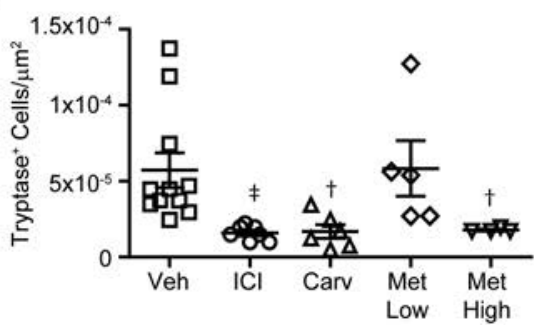

D

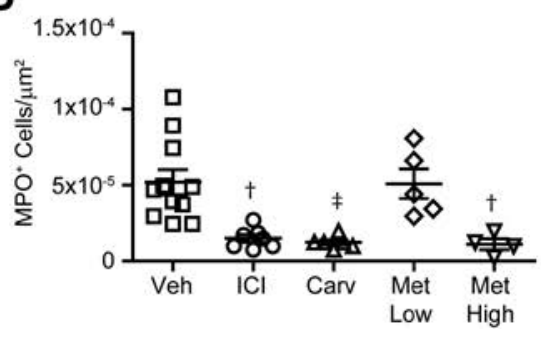

Figure 7. Chronic beta blocker infusion before MI decreases leukocyte infiltration into the heart. (A) Representative CD68, tryptase, and MPO staining (black arrowheads) within the border zone of hearts from WT C57BL/6) mice that were administered Veh, ICl, Carv, Met Low, or Met High 2 weeks before MI surgery. Scale bar: $200 \mu \mathrm{m}$. (B) Quantification of CD68 ${ }^{+}$(overall $P=0.0001$, Kruskal-Wallis test), (C) quantification of tryptase ${ }^{+}$(overall $P=0.0002$, Kruskal-Wallis test), and (D) quantification of $\mathrm{MPO}^{+}$(overall $P=0.0001$, Kruskal-Wallis test) cell infiltration into the heart from $\mathbf{A} . n=11$ for Veh, $n=7$ for ICI, $n$ $=6$ for Carv, $n=5$ for Met Low, and $n=4$ for Met High. Results of exact Wilcoxon rank-sum tests with multiple-comparison adjustment -4 comparisons, MI beta blockers versus MI Veh - are indicated in scatter dot plots. ${ }^{\dagger} P<0.01$, and ${ }^{\ddagger} P<0.001$ versus Veh.

our study (Table 1), the dose of carvedilol used in this study $(10 \mathrm{mg} / \mathrm{kg} /$ day in mice) produces a human equivalent dose of approximately $70 \mathrm{mg} /$ day, which is greater than the maximal dose used in the SELECT trial $(50 \mathrm{mg} /$ day $)(21,22)$. Similarly, the Met High dose used in our study equates to approximately 350 $\mathrm{mg} /$ day in an 85-kg human, above the maximal dose of $200 \mathrm{mg} /$ day used in the SELECT trial, while the Met Low dose produces a human equivalent dose of approximately $7 \mathrm{mg}$, well below the lowest dose of metoprolol $(25 \mathrm{mg} /$ day) used in the SELECT trial $(21,22)$. Thus, it would be expected that the doses of Carv and Met High used in our study may cause severer changes in leukocyte parameters than would be observed in humans receiving beta blockers within clinically used ranges. Indeed, our Transwell migration data are consistent with this notion because prolonged infusion with either Carv or Met High completely ablated isolated mouse leukocyte migration (no fold change in response to CCL2), whereas PBLs isolated from beta blocker-treated humans retained some responsiveness to CCL2 ( 2-fold change in migration). Therefore, although we provide a useful proof-of-concept study depicting the action of $\beta_{2}$-AR-selective beta blockers on in vivo leukocyte biology, further studies using a range of doses of clinically used beta blockers with varying $\beta_{2}$-AR selectivity will be most informative in terms of defining thresholds of molecular changes produced by chronic beta blocker treatment. In fact, titration of beta blocker dosage could be achieved in which leukocyte responsiveness is partially, but not fully, diminished to promote a more restrained innate immune response that allows for tissue repair but with reduced long-term inflammation. 
Second, beta blocker treatment that precedes an injury may dampen leukocyte responsiveness to that injury, thereby reducing the inflammatory insult and potentially relaying better outcomes. Of note, although we observed that longer beta blocker infusion regimens ( $\geq 2$ weeks) were required to significantly alter the molecular signatures of leukocytes at baseline or in response to acute cardiac injury, we did not test the impact of beta blocker treatment on leukocyte parameters for less than 1 week. To this point, work by the Ibáñez group has demonstrated a benefit of metoprolol infusion beginning just before reperfusion following MI in humans (METOCARD-CNIC trial patients) and pigs (17), which in a follow-up study was mechanistically linked with the direct negative regulation of neutrophil function and was associated with decreased myocardial injury in mice (27). Thus, the duration of beta blocker treatment necessary to dampen immune cell responses, but not negatively affect MI-induced scar formation and survival, is an open question, though this may be less of a concern than beta blocker dosage or selectivity.

Third, there is likely a critical washout period after cessation of beta blocker infusion in which full leukocyte responsiveness to acute injury may be restored. For instance, our initial experiments in which we established the impact of beta blocker infusion at baseline showed a marked increase in splenic hypertrophy and retention of leukocytes, whereas in our subsequent studies in which we discontinued beta blocker infusion 1 day before sham or MI surgery and performed cellular and tissue analyses another 4 days later ( 5 days total following beta blocker cessation), splenic parameters were almost normalized even in the sham-operated mice. In contrast, our previous work showed that chimeric mice lacking $\beta_{2}$-AR expression in cells of hematopoietic origin exhibited baseline splenomegaly that increased in conjunction with leukocyte retention following cardiac injury, with correspondingly worsened leukocyte infiltration to the heart, which was partially relieved by splenectomy (14). Although splenic parameters were normalized after beta blocker discontinuation, the beta blocker-mediated decrease in leukocyte CCR2 expression and CCL2-mediated migration following injury were retained even 5 days following cessation of infusion, indicating a prolonged impact of beta blockade on CCR2 signaling in leukocytes. This observation points out that other beta blocker-sensitive cell types, including those in the heart, may still be altered at a molecular level similar to leukocytes, thereby contributing to the outcome after MI. However, there were no differences in post-MI infarct size or cardiac function in the vehicle versus beta blocker treatment groups, suggesting that the initial response to acute MI was similar across all cohorts and that reduced immune cell infiltration into the heart by 4 days after MI was specifically due to altered leukocyte CCR2 expression and responsiveness. The intermediate phenotype of restored splenic leukocyte profile but reduced CCR2-mediated responsiveness could explain why beta blocker-treated mice experienced less mortality after MI than we previously observed with leukocyte-specific genetic ablation of $\beta_{2}$-AR (14). It also suggests that a longer washout period between beta blocker cessation and injury is required to fully recover innate immune system responsiveness to injury.

Although there are conflicting reports of the effects of the sympathetic nervous system on regulating the CCL2/CCR2 axis, with some showing no effect (28) and others showing decreased CCR2 in response to either nonselective adrenergic receptor activation (29) or beta blockade (30), previous findings in our laboratory have shown that deletion of hematopoietic $\beta_{2}$-AR almost completely ablates CCR2 expression (15). In accordance with our previous findings, this study demonstrates that chronic pharmacological inhibition in vivo, with increasing duration and selectivity toward $\beta_{2}-\mathrm{AR}$, decreases CCR2 expression in both BMCs and PBLs. Further, our clinical data highlight the translational relevance of our studies in mice because CCR2 expression and CCL2 responsiveness were also decreased in the buffy coat samples of patients having taken a beta blocker chronically versus beta blocker-naive patients. Although interpretation of human data can be made difficult by variability between patient treatment and control groups, the alterations in CCR2 expression and responsiveness we observed in human PBLs occurred irrespective of underlying conditions in the patient populations because both beta blocker-treated and beta blocker-naive cohorts comprised patients with hypertension, diabetes mellitus, coronary artery disease, or heart failure. Therefore, CCR2 expression levels in PBLs may represent a molecular biomarker for predicting the potential impact and outcomes of chronic beta blocker treatment on leukocyte-dependent responsiveness to acute injury, which will require further assessment.

Beyond use as a biomarker in this regard, CCR2 has been explored more generally as a potential therapeutic target in injury and disease models (31). Classical inflammatory monocytes express high levels of CCR2, while nonclassical reparative monocytes lack CCR2 expression (23). Previous studies have shown the importance of CCR2 in mediating classical monocyte recruitment to the heart following MI, and more recently pressure overload, and the impact of deleting CCR2 expression genetically or via siRNA on preventing this response to improve outcomes in mice $(10,24,32)$. Thus, inhibition of CCR2 remains a viable 
strategy to dampen the inflammatory response after injury, though gene deletion or siRNA strategies to alter CCR2 expression in vivo remain more challenging than a pharmacological approach. As such, selective targeting of inflammatory monocytes via beta blocker-mediated decreases in CCR2 expression may provide a strategy by which to decrease pathological remodeling following tissue injury while not interfering with reparative monocyte/macrophage processes.

In summary, our study demonstrates that clinically relevant beta blockers modulate several leukocyte parameters at a molecular level, including VCAM-1 and CCR2 expression, thereby altering their splenic localization and migration, respectively, and reducing their responsiveness to injury. The use of clinical samples in this study suggests that chronic beta blocker treatment also alters leukocyte parameters in humans. Whether this influences leukocyte responsiveness to acute injury events in humans and the subsequent remodeling outcomes remains to be tested vigorously. Thus, an expanded investigation into the impact of selectivity, dosage, and duration of beta blocker usage on human leukocyte function would offer clinical insight and drive further translational research using preclinical models.

\section{Methods}

Experimental animals. WT C57BL/6J male mice aged 10-12 weeks, obtained from The Jackson Laboratory, were used in this study to determine the impact of prior beta blocker infusion on parameters including splenic and cardiac accumulation of leukocytes before and after acute injury, alterations in expression of adhesion and chemokine receptors, as well as leukocyte chemotaxis. WT mice were randomized into groups that were administered Veh (sterile PBS with 10\% DMSO), ICI-118,551 (ICI, $0.1 \mathrm{mg} / \mathrm{kg} / \mathrm{d}$ ), metoprolol (either a low and $\beta_{1}$-AR-selective dose of $1 \mathrm{mg} / \mathrm{kg} / \mathrm{d}$, Met Low, or a high and nonselective dose of $50 \mathrm{mg} / \mathrm{kg} / \mathrm{d}$, Met High) or carvedilol (Carv, $10 \mathrm{mg} / \mathrm{kg} / \mathrm{d}$ ) via osmotic minipumps (Alzet). For acute MI experiments, infusion was discontinued 1 day before surgery to allow drug washout, and the surgeon was blinded to the treatment groups.

Coronary artery occlusion surgery. MI was induced as previously described $(14,15)$. In brief, mice were anesthetized with $2 \%$ isoflurane inhalation. A small skin incision was made, and the pectoral muscles were dissected and retracted to expose the fourth intercostal space. A small hole was made and the heart popped out. The left coronary artery was sutured approximately $3 \mathrm{~mm}$ from its origin, and the heart was placed back into the intrathoracic space. There was closure of muscle and skin. Animals received a single dose $(0.3$ $\mathrm{mg} / \mathrm{kg}$ ) of buprenorphine immediately following surgery. Injury level from MI surgery was standardized by a predefined parameter as having an ejection fraction of less than $40 \%$ as measured by echocardiography 4 days after MI, whereas animals having an ejection fraction of greater than $40 \%$ were considered as having an unsuccessful surgery and excluded from the study.

Echocardiography. Cardiac function testing was performed at baseline and at 7, 14, and 21 days after MI via transthoracic, 2-dimensional echocardiography using a VisualSonics Vevo 2100 System with a 12-MHz probe on mice anesthetized with isoflurane (1.5\%), and body temperature was maintained on a heated table with embedded ECG leads to monitor heart rate, temperature of the animal, ECG, and breathing. The parasternal short-axis views of the heart were obtained in B-mode by placing the transducer in the parasternal long axis and rotating the transducer $90^{\circ}$ clockwise to find the parasternal short axis. For measurements, M-mode echocardiography was performed in the parasternal short-axis view at the level of the papillary muscle to assess left ventricular (LV) fractional shortening and ejection fraction. Percentage of fractional shortening was calculated using the equation ([LVIDd - LVIDs]/LVIDd) $\times 100$. Percentage of ejection fraction was calculated using the equation ([LVvold - LVvols $] / L V v o l d) \times 100$. The assessor was blinded to the groups, and the data were compiled into treatment and surgical groups only after the analyses were completed. To examine the ability of low-dose metoprolol to block isoproterenol-induced cardiac contractility, echocardiography was performed following 2 weeks' administration of Veh (PBS plus 10\% DMSO) or a low dose of metoprolol (1 $\mathrm{mg} / \mathrm{kg} /$ day) at baseline and 5 minutes after $0.1 \mathrm{mg} / \mathrm{kg}$ (i.p.) isoproterenol injection.

Histological analysis. Excised hearts and spleens were fixed in 4\% paraformaldehyde, paraffin embedded, and sectioned at $5-\mu \mathrm{m}$ thickness, with the hearts subsequently stained with Masson's trichrome (MilliporeSigma). Immunohistochemistry was performed on deparaffinized sections to examine infiltration of various immune cell types as previously described $(14,15)$. Antigens were retrieved using a citrate-based Antigen Unmasking Solution (Vector Laboratories). Tissues were blocked (10\% FBS/PBS) and incubated with a $0.3 \%$ $\mathrm{H}_{2} \mathrm{O}_{2}$ solution used to block endogenous peroxide activity, followed by incubation with antibodies against CD68 (1:100; Abcam ab31360), mast cell tryptase (1:100; Abcam ab2378), or MPO (1:100, Santa Cruz). Washed slides were incubated with the appropriate secondary antibodies, anti-mouse HRP (1:1000; GE 
Healthcare) or anti-goat HRP (1:1000; Santa Cruz), followed by staining with hematoxylin. Positive controls for primary antibody detection of CD68 (spleen), tryptase (lung), and MPO (post-MI heart) and no secondary antibody control staining are shown in Supplemental Figure 6. Stained tissues were developed using a DAB Substrate Kit (Vector Laboratories) and mounted using Permount Mounting Media (Thermo Fisher Scientific). Staining was visualized on a Nikon Eclipse microscope at $\times 20$ magnification, and NIS-Elements software (Nikon) was used for recording images and image analysis. Images were quantified as the number of positive cells per area. VCAM-1 immunofluorescence was detected as previously described (14).

Immune cell isolation from the heart, spleen, BM, and blood. BMCs were isolated from the femurs of WT C57BL/6J mice as previously described $(14,15)$. PBLs were isolated from blood collected from mice using a heparinized syringe and centrifuged for 10 minutes at $94 \mathrm{~g}$. The concentrated buffy coat layer was collected, washed with PBS, and centrifuged for 5 minutes at $94 \mathrm{~g}$. De-identified human buffy coat samples were obtained from Temple University Hospital and University of Campania "Luigi Vanvitelli" from patients who were naive to beta blockers or were taking either metoprolol or carvedilol at the time of sample collection, inclusive of patients with hypertension, diabetes mellitus, coronary artery disease, or heart failure (Table 1). Mouse spleens were isolated and underwent manual digestion in PBS using a syringe plunger until a single-cell suspension was obtained. The cell slurry was then washed with PBS and centrifuged at $94 \mathrm{~g}$ for 5 minutes. For each cell preparation, the supernatant was discarded and the pellets resuspended in ammonium chloride-potassium lysis buffer to lyse red blood cells. The cells then underwent additional washes with PBS and centrifugation at $94 \mathrm{~g}$ for 5 minutes. The pellets were resuspended in PBS and strained through a primed $70-\mu \mathrm{m}$ cell strainer (VWR) before cell counting and use for RT-qPCR, cell migration, or flow cytometry analyses.

Whole hearts were isolated and flushed with HBSS to remove the blood, then manually digested into $1-\mathrm{mm}^{3}$ pieces followed by enzymatic digestion in a collagenase II $(150 \mathrm{U} / \mathrm{ml})$ and trypsin $(0.6 \mathrm{mg} / \mathrm{ml})$ solution (Worthington Biochemical). All digestion steps were carried out in a rotating $37^{\circ} \mathrm{C}$ water bath. The collagenase II/trypsin solution was replaced after each digestion, and the cell-containing solution was placed in a 50-ml Falcon tube (Thermo Fisher Scientific) with $4 \mathrm{ml}$ of FBS. Subsequent digestions were carried out until the remaining tissue pieces were too small to separate from the digestion fluid. The cell suspension was centrifuged at $845 \mathrm{~g}$ for 5 minutes, and the supernatant was discarded, after which the pellet was resuspended in $4 \mathrm{ml}$ of FBS and centrifuged at $8 \mathrm{~g}$. The nonmyocyte-containing supernatant was transferred to a new tube and centrifuged at $845 \mathrm{~g}$ for 5 minutes. The pellet was resuspended in HBSS and stained through a primed $70-\mu \mathrm{m}$ cell strainer before cell counting and use for flow cytometry analysis.

FACS analysis. Cells were stained in FACS buffer (1\% FBS in PBS) with the following antibodies: CD45BUV395 (1:100, BD Biosciences 564279), CD68-PE (1:100, BioLegend 137014), CD117-APC-H7 (1:50, BD Biosciences 560185), Ly-6G-BV421 (1:100, BD Biosciences 562737), CCR2-FITC (1:100, BioLegend 150608), and LIVE/DEAD Fixable Aqua Dead Cell Stain Kit (1:40, Invitrogen L34957). Isotype control antibodies that were used in conjunction with splenic leukocytes to validate cell staining (Supplemental Figure 7) were FITC rat IgG2b ( $\kappa$ isotype, 1:100, BioLegend 400633), APC-H7 rat IgG2b ( $\kappa$ isotype, 1:50, BD Biosciences 560200), BUV395 rat IgG2b ( $\kappa$ isotype, 1:100, BD Biosciences 563560), BV421 rat IgG2a ( $\kappa$ isotype, 1:100, BD Biosciences 562602), and PE rat IgG2a ( $\kappa$ isotype, 1:100, BioLegend 400507). Cells were stained for 30 minutes in the dark and on ice. After staining, the cells were washed twice with PBS and centrifuged for 5 minutes at $94 \mathrm{~g}$, after which the cells underwent resuspension in FACS buffer. Data were acquired on an LSRII flow cytometer and analyzed using FlowJo software (Tree Star Inc.). Splenic cells and BMCs are expressed as percentage of $\mathrm{CD}^{+} 5^{+}$cells, while cardiac cells are expressed per $1 \times 10^{6}$ cells because $\mathrm{CD} 45^{+}$cell populations differed between the Veh and ICI treatment groups in the heart only.

Migration assay. Freshly isolated BMCs or PBLs were plated on $8-\mu \mathrm{m}$ transmembrane inserts (Corning) in a 24-well plate containing Veh or CCL2 $(100 \mathrm{ng} / \mathrm{ml})$ as previously described (15). Cells were stained with Hoechst and migration was imaged after 4 hours. Cells were visualized at $\times 10$ magnification using a Nikon Eclipse microscope and analyzed from 10 random fields per treatment.

Reverse transcription quantitative PCR. cDNA was synthesized from the total RNA of spleen, BMC, or PBL samples using the High Capacity cDNA Reverse Transcription kit (Applied Biosystems). To assess CCR2 and VCAM-1 expression, RT-qPCR was performed with SYBR Select Master Mix (Applied Biosystems) in triplicate for each sample using primers listed in Supplemental Table 1 at an annealing temperature of $60.1^{\circ} \mathrm{C}$. All RT-qPCR data were analyzed using Applied Biosystems Comparative $\mathrm{C}_{\mathrm{T}}$ Method $\left(\Delta \Delta \mathrm{C}_{\mathrm{T}}\right)$. Gene expression was normalized to translationally controlled tumor protein 1, statistics were performed on $\Delta \mathrm{C}_{\mathrm{T}}$ values, and results were expressed as $2^{-\Delta \Delta \mathrm{CT}}$ or $\mathrm{RQ}$ with $\mathrm{RQ}_{\min / \max }$ indicated for range. 
Statistics. Data are presented in scatter dot plot format with mean \pm SEM indicated in all figures. Statistical comparisons of a continuous variable between different treatment groups were performed using the nonparametric Kruskal-Wallis test for 3 or more groups and exact Wilcoxon rank-sum tests with multiple pairwise comparison adjustments made using the following formula: adjusted $P$ value $=\min$ ([no. comparisons made] $\times$ [raw $P$ value], 1.00) to guard against possibly non-normally distributed data in small group sizes. Echocardiography data were analyzed using 2 -way repeated measures ANOVA. $P$ values and $n$ (group size) values are reported in the figure legends, where $P$ values less than 0.05 were considered statistically significant. All statistical analyses were performed using SAS version 9.4 software (SAS Institute Inc.). Each assay was performed in a minimum of 3 independent experiments.

Study approval. All animal procedures and experiments were carried out according to the NIH Guide for the Care and Use of Laboratory Animals (National Academies Press, 2011) and approved by the Institutional Animal Care and Use Committee of Temple University. Human buffy coat samples and data were not collected specifically for our study, and no one on our study team has access to the subject identifiers linked to the specimens or data. Thus, our study is not considered human subject research and did not require institutional review board approval.

\section{Author contributions}

LAG and DGT wrote the manuscript and designed the experiments. LAG conducted most of the experiments and analyzed the data, with contributions from TPT and AS. EG performed the sham and MI surgeries. DY performed statistical analyses. CDL, JTS, VDM, RB, CS, and RM processed human buffy coat samples or provided de-identified patient demographics. SRH, WJK, EAH, and DGT provided intellectual guidance and manuscript revision.

\section{Acknowledgments}

This work was supported by NIH grants R01 HL105414 (to DGT), R01 HL139522 (to DGT), and P01 HL091799 (to WJK and SRH) and by American Heart Association Scientific Development Grant 17SDG33400114 (to LAG) and Postdoctoral Fellowship 17POST33660942 (to CDL).

Address correspondence to: Douglas G. Tilley, Room 945A MERB, Center for Translational Medicine, Lewis Katz School of Medicine, Temple University, 3500 N. Broad St., Philadelphia, Pennsylvania 19140, USA. Phone: 215.707.9758; Email: douglas.tilley@temple.edu. Or to: Laurel A. Grisanti, University of Missouri, College of Veterinary Medicine, W191 Veterinary Medicine, 1600 E. Rollins St., Columbia, Missouri 65211, USA. Phone: 573.884.8852; Email: grisantil@missouri.edu.

1. Kratofil RM, Kubes P, Deniset JF. Monocyte conversion during inflammation and injury. Arterioscler Thromb Vasc Biol. 2017;37(1):35-42.

2. Chen B, Frangogiannis NG. Immune cells in repair of the infarcted myocardium. Microcirculation. 2017;24(1):e12305.

3. Prabhu SD, Frangogiannis NG. The biological basis for cardiac repair after myocardial infarction: from inflammation to fibrosis. Circ Res. 2016;119(1):91-112.

4. Zlatanova I, Pinto C, Silvestre JS. Immune modulation of cardiac repair and regeneration: the art of mending broken hearts Front Cardiovasc Med. 2016;3:40.

5. McDonald B, Kubes P. Innate immune cell trafficking and function during sterile inflammation of the liver. Gastroenterology. 2016;151(6):1087-1095.

6. Rao X, Zhong J, Sun Q. The heterogenic properties of monocytes/macrophages and neutrophils in inflammatory response in diabetes. Life Sci. 2014;116(2):59-66.

7. Garza MA, Wason EA, Zhang JQ. Cardiac remodeling and physical training post myocardial infarction. World J Cardiol. 2015;7(2):52-64.

8. Spahn JH, Kreisel D. Monocytes in sterile inflammation: recruitment and functional consequences. Arch Immunol Ther Exp (Warsz). 2014;62(3):187-194

9. Ismahil MA, Hamid T, Bansal SS, Patel B, Kingery JR, Prabhu SD. Remodeling of the mononuclear phagocyte network underlies chronic inflammation and disease progression in heart failure: critical importance of the cardiosplenic axis. Circ Res. 2014;114(2):266-282.

10. Majmudar MD, et al. Monocyte-directed RNAi targeting CCR2 improves infarct healing in atherosclerosis-prone mice. Circulation. 2013;127(20):2038-2046

11. Sager HB, et al. Proliferation and recruitment contribute to myocardial macrophage expansion in chronic heart failure. Circ Res. 2016;119(7):853-864.

12. Aizik G, Grad E, Golomb G. Monocyte-mediated drug delivery systems for the treatment of cardiovascular diseases. Drug Deliv Transl Res. 2018;8(4):868-882. 
13. Padro CJ, Sanders VM. Neuroendocrine regulation of inflammation. Semin Immunol. 2014;26(5):357-368.

14. Grisanti LA, et al. Leukocyte-expressed $\beta 2$-adrenergic receptors are essential for survival after acute myocardial injury. Circulation. 2016;134(2):153-167

15. Grisanti LA, Traynham CJ, Repas AA, Gao E, Koch WJ, Tilley DG. $\beta 2$-Adrenergic receptor-dependent chemokine receptor 2 expression regulates leukocyte recruitment to the heart following acute injury. Proc Natl Acad Sci U S A. 2016;113(52):15126-15131.

16. Bristow MR. Treatment of chronic heart failure with $\beta$-adrenergic receptor antagonists: a convergence of receptor pharmacology and clinical cardiology. Circ Res. 2011;109(10):1176-1194.

17. García-Ruiz JM, et al. Impact of the timing of metoprolol administration during STEMI on infarct size and ventricular function. J Am Coll Cardiol. 2016;67(18):2093-2104.

18. Bernstein D, et al. Differential cardioprotective/cardiotoxic effects mediated by $\beta$-adrenergic receptor subtypes. Am J Physiol Heart Circ Physiol. 2005;289(6):H2441-H2449.

19. Höcht C, Di Verniero C, Opezzo JA, Bramuglia GF, Taira CA. Pharmacokinetic-pharmacodynamic (PK-PD) modeling of cardiovascular effects of metoprolol in spontaneously hypertensive rats: a microdialysis study. Naunyn Schmiedebergs Arch Pharmacol. 2006;373(4):310-318

20. Lin R, et al. Changes in $\beta 2$-adrenoceptor and other signaling proteins produced by chronic administration of 'beta-blockers' in a murine asthma model. Pulm Pharmacol Ther. 2008;21(1):115-124.

21. Zebrack JS, Munger M, Macgregor J, Lombardi WL, Stoddard GP, Gilbert EM. Beta-receptor selectivity of carvedilol and metoprolol succinate in patients with heart failure (SELECT trial): a randomized dose-ranging trial. Pharmacotherapy. 2009;29(8):883-890.

22. Nair AB, Jacob S. A simple practice guide for dose conversion between animals and human. J Basic Clin Pharm. 2016;7(2):27-31.

23. França CN, et al. Monocyte subtypes and the CCR2 chemokine receptor in cardiovascular disease. Clin Sci. 2017;131(12):1215-1224.

24. Kaikita K, Hayasaki T, Okuma T, Kuziel WA, Ogawa H, Takeya M. Targeted deletion of CC chemokine receptor 2 attenuates left ventricular remodeling after experimental myocardial infarction. Am J Pathol. 2004;165(2):439-447.

25. Swirski FK, et al. Identification of splenic reservoir monocytes and their deployment to inflammatory sites. Science. 2009;325(5940):612-616.

26. Callaerts-Vegh Z, et al. Effects of different beta adrenoceptor ligands in mice with permanent occlusion of the left anterior descending coronary artery. Br J Pharmacol. 2003;138(8):1505-1516.

27. García-Prieto J, et al. Neutrophil stunning by metoprolol reduces infarct size. Nat Commun. 2017;8:14780

28. Okutsu M, Suzuki K, Ishijima T, Peake J, Higuchi M. The effects of acute exercise-induced cortisol on CCR2 expression on human monocytes. Brain Behav Immun. 2008;22(7):1066-1071.

29. Xiu F, Stanojcic M, Jeschke MG. Norepinephrine inhibits macrophage migration by decreasing CCR2 expression. PLoS One. 2013;8(7):e69167.

30. Guo YL, et al. Monocyte/macrophage $\beta 2-\mathrm{AR}$ as a target of antisympathetic excitation-induced atherosclerotic progression Genet Mol Res. 2014;13(4):8080-8088.

31. Bianconi V, Sahebkar A, Atkin SL, Pirro M. The regulation and importance of monocyte chemoattractant protein-1. Curr Opin Hematol. 2018;25(1):44-51.

32. Patel B, et al. CCR2. JACC Basic Transl Sci. 2018;3(2):230-244. 